\title{
Allergietherapie: Noch fehlen psychosomatische Konzepte
}

\begin{abstract}
Der Einfluß der Psyche ist ein wichtiger Faktor bei der Entwicklung und Aufrechterhaltung allergischer Symptome. Er führt zusammen mit äußeren Einflüssen, Lebensstil und genetischer Veranlagung auf dem Boden einer allergischen Sensibilisierung zum Ausbruch der Krankheit. Allerdings gibt es auf diesem Gebiet noch längst keine so fundierten und tragfähigen therapeutischen Konzepte wie auf der somatischen Ebene des atopischen Geschehens.
\end{abstract}

Als widerlegt, so U. Gieler (Gießen), gilt mittlerweile die Hypothese der Existenz einer typischen Allergiepersönlichkeit - gekennzeichnet durch den Begriff der ,allergischen Objektbeziehung" -, wie Ende der 50er Jahre durch den französischen Psychosomatiker Marty behauptet. „Eine dominante Mutter mag zwar im Einzelfall tatsächlich eine Rolle spielen, doch darf ein solches Beziehungsmuster nicht grundsätzlich unterstellt werden“, erklärte Gieler.

\section{Angst und Allergie eng verknüpft} Bestimmte psychische Phänomene treten bei Allergikern jedoch häufiger als in der Normalbevölkerung auf. So wird über erhöhte Aggressionswerte bzw. über eine Aggressionshemmung bei Allergikern berichtet sowie über eine erhöhte Koinzidenz von Depressionen und Allergien, wobei die beiden Krankheitsmanifestationen immer nur alternativ aufzutreten scheinen.

Speziell Urtikariapatienten weisen häufig eine Aggressionshemmung auf und können ihrem Ärger schlecht Ausdruck verleihen - bei gleichzeitig hohem Bedürfnis nach Anerkennung durch ihre Umgebung (Gupta et al. 1996). In Gielers eigener Arbeitsgruppe wurde 1993 eine psychoendokrine

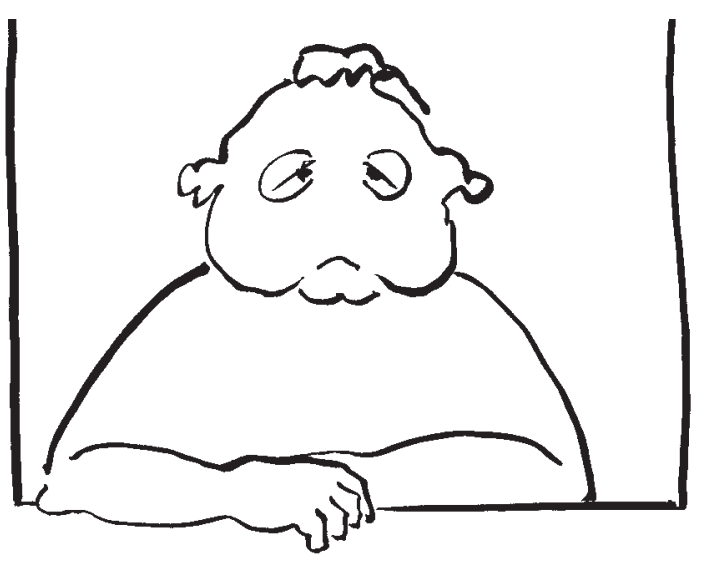

Reaktion bei Urtikariapatienten nachgewiesen, die ihre aggressiven Impulse unterdrückten.

Auch Angstsymptome sind bei Allergiepatienten überdurchschnittlich häufig zu beobachten: In einer Studie von Schmidt-Traub et al. (1995) an 100 Patienten mit einer Typ-I-Allergie wurde ein fünffach erhöhtes Risiko für die Entwicklung einer Angststörung nachgewiesen, im Vergleich zu 66 Kontrollpersonen. Rund der Hälfte der Allergiepatienten in dieser Studie mußte eine psychische Störung attestiert werden. Nach Gielers Worten läßt sich eine Allergie nicht zufriedenstellend behandeln, wenn nicht gleichzeitig gegen eine zugrundeliegende Angststörung psychotherapeutisch angegangen wird.
„Der Arzt muß jedoch im Einzelfall entscheiden, inwieweit die allergischen Symptome tatsächlich im Extremfall Ausdruck eines schweren psychischen Traumas sind oder ob lediglich die Birke vor dem Schlafzimmer als Ursache auszumachen ist", sagte Gieler. Der Allergologe sollte sich deshalb auch auf psychosomatischem Gebiet weiterbilden, damit Patienten schneller in die evtl. notwendige psychotherapeutische Behandlung überwiesen werden.

\section{Hoher Leidensdruck bei Patienten mit Nahrungsmittelallergie}

Einer kürzlich (1999) von Augustin publizierten Studie an 228 stationären Allergikern zufolge erweisen sich dabei die Patienten hinsichtlich ihrer Motivation zu psychosozialen Therapiemaßnahmen unterschiedlich aufgeschlossen: Besonders Patienten mit einer Nahrungsmittelallergie oder Urtikaria verspüren einen hohen psychischen Leidensdruck und waren einer Psychotherapie gegenüber zugänglicher als Patienten mit Insektengiftallergien oder Medikamentenunverträglichkeit.

Doch sollte man den Effekt einer psychosozialen Intervention nicht überbewerten. Gieler: „Erfahrungsgemäß bessern sich eher die subjektiven Symptome, die körperliche Symptomatik wird aber häufig nur in ihrem Verlauf gemildert." Dies mag nicht zuletzt auch daran liegen, daß die Psychosomatiker und Psychiater die Patientengruppe der Allergiker bisher eher ignoriert haben, so daß es heute noch keine brauchbaren Therapiekonzepte auf dieser Ebene gibt.

\section{Gute Erfolge mit}

\section{Streßbewältigungstherapien}

Gute Erfolge verzeichnet man heute besonders auf dem Gebiet der Streßbewältigung. Hier liefert auch die neuropsychoimmunologische Grundlagenforschung mit ihren Untersuchungen zu Neuropeptiden und Neurotrophinen eine gute Basis, die den Einfluß von Streßerleben auf die allergische Entzündungsreaktion auf naturwissenschaftlicher Basis belegen.

$b k$

U. Gieler (Gießen) anläßlich der 21. Tagung der Deutschen Gesellschaft für Allergologie und klinische Immunologie, München 1999. 